\title{
Avaliação institucional de graduados em farmácia sobre sua formação em saúde para o SUS
}

\author{
Institutional evaluation of graduates in \\ pharmacy on its health training for SUS
}

\author{
Marla Surdi ${ }^{1}$ \\ Tatiana Mezadri ${ }^{2}$ \\ Stella Maris Brum Lopes ${ }^{3}$
}

Resumo: Este trabalho teve como objetivo analisar a percepção de egressos do Curso de Farmácia de uma Universidade Comunitária de Santa Catarina sobre sua formação em saúde para o Sistema Único de Saúde (SUS). Dos 1.253 egressos 90 participaram da avaliação institucional e foram separados em três grupos: G1 que tiveram uma formação pautada no modelo biomédico, G2 que foram influenciados pelas transformações que ocorreram com a implantação das Diretrizes Curriculares Nacionais (DCN) e G3 com um currículo instituído para uma formação crítica-reflexiva voltada a atender os preceitos da saúde pública. Foi enviado por e-mail um questionário on-line com perguntas referente ao perfil sociodemográfico, profissional e formação em saúde para o SUS. Na comparação entre os Grupos foi realizada a prova não paramétrica de KrusKal-wallis seguido pelo pós-teste de Dunn. Os resultados demonstraram que a maioria dos egressos eram do sexo feminino $(80 \%)$, a faixa etária prevalente foi de 29 a 38 anos $(52,2 \%), 74,4 \%$ deram continuidade a formação após a graduação e $90 \%$ demoraram até 6 meses para ingressarem no mercado de trabalho. A percepção dos egressos com relação a participação durante sua formação em atividades referentes a formação para o SUS demostraram resultados significativos $(\mathrm{p}<0,05)$ para todas as variáveis com diferença expressiva antes das DCN e da implantação do Programa de Reorientação da Formação Profissional para o SUS (Grupo 1) e após (Grupos 2 e 3). Conclui-se que, na percepção dos egressos, o curso contribuiu para uma formação com abordagem integral do processo saúde-doença com ênfase na atenção básica, promovendo transformações no ensino e aprendizagem e de prestação de serviços à comunidade.

Palavras-chave: Educação em Farmácia. Formação profissional. Sistema Único de Saúde. 
Abstract: This study aimed to analyze the perception of graduates of the Pharmacy Course at a Community University in Santa Catarina about their health education for the Unified Health System (SUS). Of the 1,253 graduates, 90 participated in the institutional assessment and were separated into three groups: G1 who had a training based on the biomedical model, G2 who were influenced by the changes that occurred with the implementation of the National Curricular Guidelines (DCN) and G3 with a curriculum instituted for a critical-reflexive formation aimed at meeting the precepts of public health. An online questionnaire was sent by email with questions regarding the socio-demographic, professional and health education profile for SUS. In the comparison between the Groups, the KrusKalwallis non-parametric test was performed followed by the Dunn post-test. The results showed that the majority of the graduates were female (80\%), the prevalent age group was 29 to 38 years old. (52.2\%), $74.4 \%$ continued their training after graduation and $90 \%$ took up to 6 months to enter the job market. The graduates' perception of participation during their training in activities related to training for SUS showed significant results $(\mathrm{p}<0.05)$ for all variables with significant difference before the DCN and the implementation of the Professional Training Reorientation Program for SUS (Group 1) and after (Groups 2 and 3). It is concluded that, in the perception of the graduates, the course contributed to a training with a comprehensive approach to the health-disease process with an emphasis on primary care, promoting changes in teaching and learning and the provision of services to the community.

Keywords: Pharmacy education. Professional qualification. Health Unic System.

\footnotetext{
${ }^{1}$ Universidade do Vale do Itajaí | Programa de Mestrado Profissional em Saúde e Gestão do Trabalho | Itajaí | SC | Brasil. Contato: marla@univali.br. ORCID: 0000-0003-0043-1479

${ }^{2}$ Universidade do Vale do Itajaí| Programa de Mestrado Profissional em Saúde e Gestão do Trabalho | Itajaí | SC | Brasil. Contato: mezadri@univali.br. ORCID: 0000-0001-7889-7936

${ }^{3}$ Universidade do Vale do Itajaí| Programa de Mestrado Profissional em Saúde e Gestão do Trabalho | Itajaí | SC | Brasil. Contato: stella@univali.br. ORCID: 0000-0001-6314-7294
}

- Recebido em: 7 de novembro de 2020

- Aprovado em: 24 de junho de 2021

DOI: http://dx.doi.org/10.1590/S1414-40772021000300012

Este é um artigo publicado em acesso aberto sob uma licença Creative Commons https://creativecommons.org/licenses/by-nc/4.0/ 


\section{Introdução}

A história da farmácia e da profissão farmacêutica é muito antiga e ao longo do tempo passou por inúmeras transformações, crises, mudanças e evoluções, atravessando momentos políticos, econômicos e sociais importantes (SERAFIN; CORREIA JÚNIOR; VARGAS, 2015).

Na década 80, com as mudanças e transformações na área da saúde provocadas pela Reforma Sanitária e concretização do Sistema Único de Saúde (SUS), surge uma nova necessidade de Reforma na Educação que implicaria em trabalho articulado entre o SUS e as instituições formadoras (FARHAT et al., 2016), pois sua efetivação dependia de reformas no sistema acadêmico. Diante deste panorama, houve a necessidade de uma reorganização do currículo dos Cursos da área da saúde, incluindo o Curso de Farmácia, delineando um novo perfil para os profissionais farmacêuticos (DOURADO; COELHO, 2010) e culminando com a criação das novas Diretrizes Curriculares Nacionais - DCN (CNE/CES 2, de 19 de fevereiro de 2002) (CNE, 2002).

As DCN apontam para um profissional que tenha um perfil multiprofissional e multidisciplinar, conforme preceitos do SUS e estabelece as competências e habilidades necessárias para o exercício da profissão: atenção à saúde, tomada de decisão, comunicação, liderança, administração e gerenciamento e educação permanente (SPADA; CHAGAS; SILVA; CASTILHO, 2006).

Aliado as DCN, o Ministério da Saúde em parceria com o Ministério da Educação, propõe uma nova formação em saúde e criam o Programa Nacional de Reorientação da Formação Profissional (Pró-Saúde), e posteriormente o Programa de Educação pelo Trabalho para Saúde (Pet-Saúde), que foram aprovados pelo Conselho Nacional de Saúde (CNS). Esses programas têm um objetivo comum que é a integração ensino-serviço-comunidade, promovendo transformações no processo de formação, geração de conhecimento e na prestação de serviços à população de forma integral no processo saúde-doença (BRASIL, 2007). O Curso de Farmácia da Universidade do Vale do Itajaí (Itajaí/SC) participou ativamente desses projetos desde 2007, promovendo aos futuros farmacêuticos a possibilidade de desempenharem um papel importante no cuidado integral da saúde da família e da comunidade (FARHAT et al., 2016).

Após mais de uma década de constantes esforços da universidade para atingir uma formação em saúde de acordo com as perspectivas do SUS, surgiu a necessidade de mesurar os resultados utilizando como instrumento metodológico a avaliação institucional, a qual é essencial para a busca da qualidade e eficiência na educação superior, além de confrontar as 
competências desenvolvidas durante o curso com as requeridas no exercício profissional. E a pesquisa com egressos proporciona a Instituição de Ensino Superior (IES) refletir sobre os desafios e fragilidades da formação, podendo assim, traçar metas e planejamentos para implementar reformas educacionais quando necessárias (LIMA; ANDRIOLA, 2018; CALBINO; CASTRO; GONÇALVES; SABINO, 2020).

\section{Formação em saúde para o SUS}

A formação em saúde está relacionada com a transformação das práticas profissionais, devido aos movimentos políticos e sociais que levaram a criação do SUS, que tem como princípios a equidade, a integralidade e a universalidade. Essa transformação proporciona uma atuação diferenciada sobre o processo saúde-doença (BATISTA; GONÇALVES, 2011).

Um dos pilares da formação em saúde é o princípio da integralidade que tem como definição na Constituição Federal de 1988 a necessidade de compreender o indivíduo enquanto um todo holístico, um ser biopsicossocial em sua essência (BRASIL, 1988). A prática da integralidade é discutida por autores da área da saúde como uma estratégia para a concretização de uma assistência voltada para as reais necessidades da população, significando um olhar mais ampliado sobre as questões biológicas, as diferentes realidades que envolvem essas pessoas, tanto na área psíquica, quanto social e regional (CECCIM; FEUERWERKER, 2004).

O desafio das instituições formadoras segundo Viana et al. (2018, p. 15) "é substituir o modelo tradicional de formação, baseado na organização do cuidado em saúde, centrado na doença e no atendimento hospitalar por um modelo de abordagem pedagógica, que considere a integralidade do processo saúde-doença".

Preparar os profissionais para implementar um novo modelo pedagógico onde o trabalho em equipe multiprofissional, a atuação interdisciplinar e o cuidado integral, são cada vez mais pré-requisitos para atuar nos diferentes níveis do sistema de saúde, em especial a Atenção Básica (AB), conforme preconizam as DCN ao presumir que a formação deve acompanhar o sistema de saúde vigente no país e dar ênfase na atenção integral à saúde, não é uma tarefa fácil para as instituições formadoras (RECINE et al., 2018).

Também pode-se observar que novos passos, para além das DCN, são necessários para que as mudanças na formação ocorram amplamente nos cursos de graduação em saúde (CECCIM; FEUERWERKER, 2004).

Com relação a formação farmacêutica, é preciso graduar um profissional que possa conduzir de forma única e autônoma seu processo de aprendizagem profissional, que seja capaz de se moldar de acordo com as mudanças que a profissão possa exigir, que seja crítico em suas 
decisões, que seja acima de tudo humano e tenha uma relação ética com a profissão e com seus pacientes (PEREIRA; FREITAS, 2008).

\section{Metodologia}

Esta pesquisa é de abordagem quantitativa, de corte transversal do tipo exploratório e foi aprovada pelo Comitê de Ética em Pesquisa da Universidade do Vale do Itajaí - UNIVALI (CAAE: 14190619.4.0000.0120). A população deste estudo foram todos os egressos $(n=1253)$ do Curso de Farmácia desta instituição, desde sua implantação em 1993 até o ano de 2018. Como critério de inclusão, o egresso deveria ter concluído a graduação no Curso de Farmácia da UNIVALI. Os dados foram coletados entre os meses de agosto a novembro de 2019.

Foi utilizado para coleta das informações um questionário on-line construído por meio da plataforma Google-forms, padronizado e elaborado especificamente para essa pesquisa. Este instrumento foi composto por questões fechadas construídas a partir do Projeto Pedagógico do Curso de Farmácia (2016-2017), das Diretrizes Curriculares Nacionais do Curso de Farmácia de 2002 e dos editais interministeriais que contemplam os programas de Reorientação da Formação Profissional para Área da Saúde o Pró e Pet-Saúde.

O questionário comtemplava informações sociodemográficas, formação acadêmica e formação para o SUS, sendo as respostas compostas por uma escala de evidências em que "1" representava nenhuma evidência e "10" evidência máxima. Para fins de análise e discussão, foi considerado um valor de percepção na escala apresentada de 5 e 6 como na "média", 7 e 8 como "bom" e 9 e 10 sendo "ótimo".

O link para preenchimento do questionário foi enviado pela coordenação do Curso de Farmácia para a lista de e-mails de egressos quatro vezes não consecutivas, durante o período proposto e conforme à queda no número diário de respostas, além disso, os egressos foram convidados pelas redes sociais (Instagram) e aplicativo telefônico (WhatsApp). Também foram enviadas as orientações para o preenchimento do questionário juntamente com o Termo de Consentimento Livre e Esclarecido (TCLE).

Para análise das respostas constituiu-se três grupos: de 1996 a 2002 - Grupo 1, de 2003 a 2009 - Grupo 2 e de 2010 a 2018 - Grupo 3; de acordo com as alterações nas diretrizes curriculares e início dos programas de reorientação da formação profissional para área da saúde o Pró e Pet-Saúde.

Os egressos que constituíram o Grupo 1 (G1) tiveram uma formação voltada ao medicamento e a prática hospitalar (Modelo Biomédico), sendo o ensino voltado para habilidades mais tecnológicas. O Grupo 2 (G2) já começava a sofrer influências das 
transformações que ocorreram com a implantação das Diretrizes Curriculares Nacionais, publicada pela Resolução CNE/CES 2, de 19 de fevereiro de 2002, com um perfil multiprofissional e multidisciplinar, conforme preceitos do SUS, e estabelece as competências e habilidades necessárias para o exercício da profissão: atenção à saúde, tomada de decisões, comunicação, liderança, administração e gerenciamento e educação permanente (SPADA; CHAGAS; SILVA; CASTILHO, 2006). Os egressos mais recentes, Grupo 3 (G3), tinham instituído em seu currículo uma formação crítica-reflexiva voltada para atender os preceitos do SUS, esses alunos participaram ativamente de estágios e de trabalhos de extensão favorecendo uma integração com o sistema local e regional de saúde.

Para a análise descritiva dos dados foram utilizados os valores absoluto, relativo e mediana. Considerando a natureza não normal das variáveis, na comparação entre os Grupos foi realizada a prova não paramétrica de KrusKal-wallis seguido pelo pós-teste de Dunn, com auxílio do programa Statistica versão 10.0. As diferenças foram consideradas significativas quando $\mathrm{p}<0,05$.

\section{Análise e discussão dos resultados}

\subsection{Caracterização Sociodemográfica}

Dos 1.253 egressos convidados para participar da pesquisa, $97(7,7 \%)$ responderam o questionário, no entanto, sete não foram elegíveis para este estudo por não terem concluído o Curso de Graduação em Farmácia na UNIVALI. De acordo com os critérios estabelecidos, o Grupo 1 foi formado por 14 (15,5\%) ex-alunos, o Grupo 2 por $30(33,3 \%)$ e o Grupo 3 por 46 $(51,2 \%)$ egressos, sendo que alguns anos ficaram sem representação no estudo.

Constatou-se nesta avaliação institucional, que a maioria dos egressos do Curso de Farmácia eram do sexo feminino (80\%), que a faixa etária prevalente foi de 29 a 38 anos $(52,2 \%)$ e $93,3 \%$ residiam em Santa Catarina. Dos participantes, 92,2\% atuavam em áreas farmacêuticas e relataram uma renda mensal acima de $\mathrm{R} \$ 5.000,00$ (52,2\%). A nível de aprimoramento profissional, observou-se que 74,4\% deram continuidade a formação após a graduação e 90\% demoraram até 6 meses para ingressarem no mercado de trabalho farmacêutico (Tabela 1). 
Tabela 1 - Caracterização de egressos do Curso de Farmácia $(n=90)$ de uma Universidade Comunitária de Santa Catarina. Itajaí (SC)

\begin{tabular}{|c|c|c|}
\hline CARACTERÍSTICAS & $\mathrm{N}$ & $\%$ \\
\hline \multicolumn{3}{|l|}{ Sexo } \\
\hline Feminino & 72 & 80,0 \\
\hline Masculino & 18 & 20,0 \\
\hline \multicolumn{3}{|l|}{ Faixa etária } \\
\hline $19-28$ & 25 & 27,8 \\
\hline $29-38$ & 47 & 52,2 \\
\hline $39-58$ & 18 & 20,0 \\
\hline \multicolumn{3}{|l|}{ Local de residência } \\
\hline Santa Catarina & 84 & 93,3 \\
\hline Outros & 6 & 6,7 \\
\hline \multicolumn{3}{|l|}{ Atuação em áreas farmacêuticas } \\
\hline Sim & 83 & 92,2 \\
\hline Não & 7 & 7,8 \\
\hline \multicolumn{3}{|l|}{ Renda } \\
\hline Até $\mathrm{R} \$ 3.000$ & 15 & 16,7 \\
\hline De $R \$ 3000$ a $R \$ 5.000$ & 28 & 31,1 \\
\hline Acima de $\mathrm{R} \$ 5.000$ & 47 & 52,2 \\
\hline \multicolumn{3}{|l|}{ Aprimoramento profissional } \\
\hline Especialização & 40 & 44,4 \\
\hline Mestrado & 22 & 24,4 \\
\hline Doutorado & 5 & 5,6 \\
\hline Não realizou aprimoramento profissional & 23 & 25,6 \\
\hline \multicolumn{3}{|c|}{ Tempo referente ao primeiro emprego na área farmacêutica } \\
\hline Até 6 meses & 81 & 90,0 \\
\hline De 6 meses a 2 anos & 9 & 10,0 \\
\hline
\end{tabular}

O percentual de respostas ser maioritariamente do sexo feminino pode estar relacionado a "feminização" da profisssão farmacêutica e ocupações em saúde, ou ainda, o tema pode ter sido mais atrativo às profissionais mulheres, conforme estudos vêm demonstrando (FRANÇA FILHO et al., 2008; SERAFIN; CORREIA JÚNIOR; VARGAS, 2015; OLIVEIRA; SZABO; BASTOS; PAIVA, 2017; MATOS; TOASSI; OLIVEIRA, 2013).

Barros, Lima, Rocha e Silva (2013) comentam que a maioria dos egressos de cursos de Farmácia seguem a carreira profissional em diferentes áreas farmacêuticas, pois o mercado de trabalho é próspero e o constante crescimento da indústria farmacêutica e cosmética nos últimos anos contribuiu bastante para melhorar as perspectivas em termos de empregabilidade deste profissional, o que corrobora com o presente estudo, em que 92,2\% dos egressos seguem em áreas farmacêuticas. 
Quanto a remuneração média do farmacêutico, segundo o Sindicato dos Farmacêuticos de Santa Catarina (SC), o piso salarial para a região do Vale do Itajaí é de R\$ 4.052,88 e para as demais regiões do estado de SC é de R \$ 3.351,42 para o ano de 2020 e observa-se que 52,2\% dos egressos do Curso de Farmácia da UNIVALI estão recebendo acima do mínimo proposto sindicalmente, diferente do estudo de Araújo (2016), o qual encontrou que a média de remuneração foi de R \$ 3.542,31 para profissionais farmacêuticos em São Paulo (SP), podendo variar de acordo com a região desse Estado. Embora o piso salarial assegure a remuneração base para o desempenho das atividades propostas e para valorização profissional, vale enfatizar que os farmacêuticos não possuem salários profissionais únicos em todo Brasil, ou seja, cada Estado tem o seu piso estipulado pelo correspondente sindicato ou pelas convenções e acordos coletivos firmados (SILVA; TORMIN; PAULA, 2019), o que pode justificar a diferença entre os pisos de SC e SP. Até aqui

O egresso recém-formado busca ascensão profissional e econômica muitas vezes optando pela continuidade dos estudos e qualificação profissional após a graduação, como demonstra a pesquisa publicada em 2015 pelo Conselho Federal de Farmácia, em que 67,5\% dos profissionais entrevistados buscaram algum tipo de especialização, pois, além da formação generalista que demanda a necessidade de uma qualificação, a farmácia possui mais de 135 especialidades distribuídas em 10 áreas de atuação que são regulamentadas pela Resolução/CFF $n^{\circ} 572 / 2013$ (CFF, 2013).

Barros, Lima, Rocha e Silva (2013) também comprovam este dado em seu estudo, com $65 \%$ dos egressos afirmando terem concluído algum tipo de qualificação. Ressalta-se ainda que, a atualização profissional na área da saúde se faz necessária, em sua maioria, pois vivemos em uma sociedade que está em constante evolução e, por isso, é importante interagir com as necessidades e os desenvolvimentos nas áreas científicas e tecnológicas da atualidade.

No que se refere a inserção no mercado de trabalho farmacêutico $90 \%$ dos egressos deste estudo relataram o início da atuação profissional em até 6 meses após a conclusão da graduação, assim como no trabalho científico de Silva et al. (2019) que encontraram resultados positivos sobre a inserção profissional, pois seus dados revelaram que 78,95\% ingressaram de maneira imediata após a formatura e 10,53\% em menos de 6 meses. Este fato pode estar relacionado a Lei $\mathrm{n}^{\mathrm{o}}$ 13.021/14 que transformou a farmácia no Brasil num estabelecimento de prestação de assistência farmacêutica, assistência à saúde e orientação sanitária individual ou coletiva, proporcionando uma maior inserção dos farmacêuticos no mercado de trabalho. 


\subsection{Formação para o SUS}

A Tabela 2 apresenta a percepção dos egressos do Curso de Farmácia com relação a participação durante sua formação em atividades referentes a formação para o SUS. Os resultados foram significativos $(\mathrm{p}<0,05)$ para todas as variáveis com diferença expressiva antes das Diretrizes Curriculares Nacionais e da implantação do Programa de Reorientação da Formação Profissional para o SUS (Grupo 1) e após (Grupos 2 e 3).

Tabela 2 - Mediana da percepção dos egressos do Curso de Farmácia $(n=90)$ de uma Universidade Comunitária com relação ao grau de participação durante sua graduação em atividades referentes a formação para o Sistema Único de Saúde. Itajaí (SC), 2020

\begin{tabular}{lcccr}
\hline \multicolumn{1}{c}{ VARIÁVEL } & $\begin{array}{c}\text { Grupo 1 } \\
\text { Mediana }\end{array}$ & $\begin{array}{c}\text { Grupo 2 } \\
\text { Mediana }\end{array}$ & $\begin{array}{c}\text { Grupo 3 } \\
\text { Mediana }\end{array}$ \\
\hline Ensino serviço comunidade & $4,5^{\mathrm{a}}$ & $7^{\mathrm{b}}$ & $8^{\mathrm{c}}$ & 0,0000 \\
\hline Seminários com dados reais da comunidade & $4,5^{\mathrm{a}}$ & $5^{\mathrm{b}}$ & $7^{\mathrm{c}}$ & 0,0005 \\
\hline Multidisciplinaridade & $2,5^{\mathrm{a}}$ & $5,5^{\mathrm{b}}$ & $7^{\mathrm{c}}$ & 0,0006 \\
\hline Visitas técnicas nos campos de atuação profissional & $4,5^{\mathrm{a}}$ & $5,5^{\mathrm{b}}$ & $8^{\mathrm{c}}$ & 0,0003 \\
\hline Acompanhamento junto equipe multiprofissional & $2^{\mathrm{a}}$ & $3^{\mathrm{a}}$ & $6^{\mathrm{b}}$ & 0,0142 \\
\hline Prática Hospitalar & $4^{\mathrm{a}}$ & $6,5^{\mathrm{b}}$ & $7^{\mathrm{c}}$ & 0,0120 \\
\hline
\end{tabular}

Nota: Grupo 1 - 1996 a 2002 (n=14); Grupo 2 - 2003 a $2009(n=30)$ e Grupo 3 - 2010 a 2018 (n=46). Letras diferentes nas linhas representam diferença estatística entre os grupos com $\mathrm{p}<0,05$ (teste de KrusKal-wallis seguido pelo pós-teste de Dunn).

O Governo Federal vem implementando políticas de inclusão social que tem expressões concretas nas áreas sociais, especialmente na Saúde e na Educação. Esta aproximação estratégica entre estas áreas, deu início a qualificação da formação profissional em saúde (DIAS; LIMA; TEIXEIRA, 2013).

Os programas instituídos Programa Nacional de Reorientação da Formação Profissional em Saúde (PRÓ-SAÚDE) e Programa de Educação para o Trabalho para a Saúde (PETSAÚDE) compõem um conjunto de políticas indutoras da formação profissional em saúde, cuja intenção é deslocar o eixo da formação centrada no modelo biologista para uma formação que considere a integralidade da atenção em saúde, comprometida com os princípios e diretrizes do Sistema Único de Saúde (SUS). Com este propósito os Programas tiveram como imagemobjetivo mudanças na formação do profissional e para tal ancoram-se nas Diretrizes Curriculares Nacionais para os cursos de saúde (BATISTA et al., 2015).

O Programa Nacional de Reorientação da Formação Profissional em Saúde (BRASIL, 2009), propõe três eixos para o seu desenvolvimento, são eles: a Orientação Teórica, Cenários de Prática e Orientação Pedagógica, onde os egressos do Curso de Farmácia dos Grupos 2 e 3 demonstraram ter uma maior percepção dessas atividades, principalmente no Eixo Cenários de 
Práticas, pois estes programas iniciaram na Universidade a partir do ano de 2005, sendo assim, estes Grupos participaram e perceberam a movimentação referente aos cenários de práticas relacionadas ao SUS desde os primeiros períodos.

Segundo o Programa de Reorientação da Formação Profissional em Saúde no eixo que propõe os Cenários de Práticas, é necessário que ocorra uma interação do aluno com a população e com os profissionais de saúde desde o início do processo de formação. Silva, Miguel e Teixeira (2011) em seu estudo, mostra que esta interação entre os três atores envolvidos, no início do processo de formação, incorpora novos conhecimentos, desenvolvimento de novas habilidades e atitudes preconizadas pelas DCN e o Curso de Farmácia, segundo o PPC 2016-2017, proporciona essa vivência e esses conhecimentos desde seus primeiros períodos aos acadêmicos, comprovando uma percepção maior dos mesmos nessa variável conforme os grupos mais recentes, que participaram de práticas em ambulatórios e atividades junto à comunidade.

A participação de estudantes de farmácia nas atividades do programa PET-SAÚDE, tem contribuído para a formação de um profissional farmacêutico generalista, humanista, crítico e reflexivo. Os resultados destes investimentos interministeriais evidenciam melhorias na qualificação dos estudantes e por consequência das práticas de saúde, visto que os futuros profissionais se sentem mais preparados para atenderem as necessidades da sociedade; com a construção de novos currículos e consolidação da integração ensino-serviço (BATISTA et al., 2015).

Os resultados apontam que a variável ensino, serviço e comunidade apresenta uma diferença significativa crescente entre os grupos. A comparação do Grupo 1 com os demais grupos demostra que esta variável nestes anos de formação predomina o conceito de saúde e doença com uma formação voltada ao modelo biomédico, sem cenários de práticas em consonância com a realidade do município. Novamente os Programas (Pro-saúde e Pet-saúde) trazem os cenários de práticas e qualificam a formação do profissional farmacêutico trabalhando a relação com a teoria da concepção de saúde-doença e a prática aplicada, nos Grupo 2 e 3 este resultado é significativo.

As variáveis relacionadas aos seminários com dados reais da comunidade e visitas técnicas nos campos de atuação profissional, possuem significados diferentes, mas os resultados foram semelhantes nos Grupos 2 e 3. Estas variáveis estão diretamente relacionadas com o conteúdo teórico a ser trabalhado com relação a prática, considerando os Grupos 2 e 3 uma formação generalista, os determinantes sociais e biológicos, diversificação e qualidade dos cenários de práticas e a integração básico-clínico, estes itens podem corresponder as variáveis. 
Os eixos propostos pelos programas buscam envolver ações no qual o eixo ético/epistemológico vislumbra fomentar mudanças na prática pedagógica relativas à orientação teórica, cenários de prática e na orientação pedagógica, assegurando uma abordagem integral do processo saúdedoença, com ênfase na atenção básica, promovendo transformações nos processos de geração de conhecimentos, ensino e aprendizagem e prestação de serviços à população.

Cada vez mais, vem crescendo o debate sobre a formação dos profissionais de saúde, e a necessidade de um novo paradigma, pensado na perspectiva de uma nova forma de produzir saúde. Diante disso, o resultado da variável multidisciplinaridade aponta que os Grupos 2 e 3 estão na crescente desta variável. No Sistema Único de Saúde a intenção é contribuir com a qualidade de vida do indivíduo, família, comunidade, em uma interpretação mais complexa dos fenômenos da saúde e da doença pela multidisciplinaridade do conhecimento, intersetorialidade das práticas e integralidade da atenção à saúde (BRASIL, 2009) justificando com isso os resultados apresentados, em comparação ao Grupo 1 que deve ter tido menos oportunidade de vivenciar o Sistema Único de Saúde.

A necessidade da formação profissional articulada entre o SUS e a Instituição de Ensino para fomentar a organização da integração ensino/serviço/comunidade, envolvendo os atores do SUS, comunidade acadêmica, gestores e usuários com foco na interprofissionalidade, interdisciplinaridade, intersetorialidade e trabalho em redes (BRASIL, 2009). Isso resulta na variável relacionada ao acompanhamento junto equipe multiprofissional. O resultado para esta variável aponta uma significativa crescente somente ao Grupo 3, que teve participação ativa no programa Pro-Pet. Um desafio, entender e colocar em prática os termos, interdisciplinar, multiprofissional, multidisciplinar, entre outros. Todos usados para a interação de campos de conhecimento e núcleos profissionais voltados a qualidade do cuidado, entre outras palavras, é aprender sobre o outro, com o outro, e entre si, para uma atuação integrada em equipe, na qual a colaboração e o reconhecimento da interdependência das áreas predominem frente à competição e a fragmentação histórica da formação uniprofissional (PEDUZZI; AGRELI, 2018).

Por fim, destaca-se que a variável Prática Hospitalar aumentou significativamente na percepção dos egressos com relação ao grau de participação durante sua graduação em atividades referentes a formação para o SUS, pois ainda que esta prática acompanhe o curso desde sua implantação, o modelo não é mais o mesmo, onde se tinha (G1) uma visão curativa, hospitalocêntrica, focada na doença e desarticulada do sistema público (BRASIL, 2009). Após a implantação dos Programas Pró e Pet-Saúde os alunos dos Grupos 2 e 3 vivenciaram as 
atividades da farmácia hospitalar nos cenários de prática com ênfase em uma abordagem de saúde integral e interdisciplinar.

Como limitações deste estudo, encontra-se o baixo número de respostas aos questionários e consequentemente alguns anos ficaram sem representação. Embora, a pesquisa on-line é um método válido e com inúmeros pontos positivos, como o alcance global, a flexibilidade para responder em qualquer horário ou local desejado, a facilidade de coleta e tabulação dos dados, o baixo custo e a facilidade de administração da pesquisa (GONÇALVES, 2008). É válido considerar ainda que este estudo aborda a avaliação institucional da prática de um curso de uma instituição de ensino superior comunitária, o que não reflete a formação de outros cursos de graduação em farmácia, ainda que tenham participado das mesmas influências curriculares baseadas nas DCN e dos editais de reorientação da formação profissional para o SUS.

\section{Conclusão}

A profissão farmacêutica encontra-se em constante evolução e com isso busca resgatar, em sua essência, o cuidado com o outro, que foi se perdendo ao longo dos anos, devido as mudanças políticas e sociais e o crescimento industrial.

Os resultados da avaliação institucional com os egressos do Curso de Farmácia da UNIVALI, apontaram para um perfil constituído em sua maioria de mulheres, com a idade variando entre 29 e 38 anos, residentes no Estado de Santa Catarina, atuantes nas áreas farmacêuticas, que deram continuidade ao aprimoramento profissional e demoraram menos de 6 meses para ingressarem no mercado de trabalho. Salienta-se que conhecer o perfil do egresso reflete para o Curso de Graduação uma importante ferramenta de atualização, planejamento e desenvolvimento de ações que possam preencher lacunas que foram deixadas durante a formação, visando melhorar o desempenho profissional.

As contribuições das ações decorrentes das Diretrizes Curriculares Nacionais aliadas aos Programas de Reorientação da Formação para o SUS no perfil dos egressos, foram significativamente mais evidenciadas no Grupo 3, reconhecendo os esforços das ações instituídas entre a universidade e as equipes de saúde do município quanto a formação generalista crítica e reflexiva que a profissão requer.

Muitos desafios ainda estão previstos nesse horizonte da formação Farmacêutica, pois mesmo que haja espaço adequado para o fortalecimento da formação em saúde nas políticas existentes, sempre haverá a necessidade da formação profissional articulada entre o SUS e a 
Instituição de Ensino Superior para fomentar essa organização entre ensino/serviço/comunidade.

Por fim, sugere-se que esta pesquisa seja reproduzida em outras universidades com características semelhantes à deste estudo a fim de conhecer o perfil do egresso e monitorar os impactos das ações e dos esforços financeiros entre Universidade e Ministérios da Saúde e da Educação e Municípios para a transformação da formação em saúde para o SUS.

\section{Referências}

ARAÚJO, Ana Luiza Lima. Percepção de farmacêuticos e graduandos sobre o curso de farmácia e a inserção no mercado farmacêutico na grande São Paulo. Orientadora: Simone Aquino. 2016. 89 f. Dissertação (Mestrado Profissional em Administração - Gestão em Sistemas de Saúde) - Universidade Nove de Julho, São Paulo, 2016. Disponível em: http:// hdl.handle.net/123456789/1239. Acesso em: 22 jun. 2020.

BARROS, Lucas Cavalcante Farias de; LIMA, Thassia Silva de Andrade; ROCHA, Thiago José Matos; SILVA, Edriane Teixeira da. Perfil do egresso do curso de farmácia de uma instituição particular do município de Maceió-AL. Revista Eletrônica de Farmácia, Goiânia, v. 10, n. 4, p. 1-15, 2013. Disponível em: https://www.revistas.ufg.br/REF/article/view/22145/15744. Acesso em: 09 mar. 2019.

BATISTA, Karina Barros Calife; GONÇALVES, Otília Simões Janeiro. Formação dos profissionais de saúde para o SUS: significado e cuidado. Saúde Soc., São Paulo, v. 20, n. 4, p. 884-889, out./dez. 2011. Disponível em:

http://www.scielo.br/scielo.php?script=sci_arttext\&pid=S0104-12902011000400007. Acesso em: 9 maio 2019.

BATISTA, Sylvia Helena Souza da Silva et al. Formação em Saúde: reflexões a partir dos Programas Pró-Saúde e PET-Saúde. Interface - Comunicação, Saúde, Educação, Botucatu, v. 19, suppl 1, p. 743-752, 2015. Disponível em: https://www.scielo.br/pdf/icse/v19s1/18075762-icse-19-s1-0743.pdf. Acesso em: 28 fev. 2019.

BRASIL. Constituição (1988). Constituição da República Federativa do Brasil. Brasília: Senado Federal, 1988.

BRASIL. Ministério da Saúde. Portaria Interministerial no 3.019, de 26 de novembro de 2007. Dispõe sobre o Programa Nacional de Reorientação da Formação Profissional em Saúde-Pró-Saúde para os cursos de graduação da área da saúde. Brasília: Ministério da Saúde, 2007.

BRASIL. Ministério da Saúde. Ministério da Educação. Programa Nacional de

Reorientação da Formação Profissional em Saúde - Pró-Saúde: objetivos, implementação e desenvolvimento potencial. Brasília: Ministério da Saúde, 2009. (Série C. Projetos, Programas e Relatórios).

CALBINO, Daniel; CASTRO, Priscila da Silva; GONÇALVES, Edinalva Rodrigues; SABINO, Geruza Tomé. Avaliação dos egressos de engenharias: um estudo a partir da inserção e desafios no mercado das primeiras turmas da UFSJ (2013-2017). Avaliação, Campinas; Sorocaba, v. 25, n. 2, p. 477-500, 2020. Disponível em:

https://www.scielo.br/j/aval/a/pvdmTNtRtmnHzTj375Xjsxd/?lang=pt. Acesso em: 28 out. 2020. 
CECCIM, Ricardo Burg; FEUERWERKER, Laura C. Macruz. Mudança na graduação das profissões de saúde sob o eixo da integralidade. Caderno de Saúde Pública, Rio de Janeiro, v. 20, n. 5, p. 1400-1410, 2004. Disponível em: http://www.scielo.br/pdf/csp/v20n5/36.pdf. Acesso em: 2 maio 2019.

CFF - CONSELHO FEDERAL DE FARMÁCIA. Resolução no 572, de 25 de abril de 2013. Dispõe sobre a regulamentação das especialidades farmacêuticas, por linhas de atuação. 2013. Disponível em: http://www.cff.org.br/userfiles/file/resolucoes/572.pdf. Acesso em: 06 mar. 2019.

CNE - CONSELHO NACIONAL DE EDUCAÇÃO. Câmara de Educação Superior. Resolução CNE/CES no 2, de 19 de fevereiro de 2002. Diretrizes Curriculares Nacionais do Curso de Graduação em Farmácia e dá outras providências. Brasília: MEC, 2002. Disponível em: http://portal.mec.gov.br/cne/arquivos/pdf/CES022002.pdf. Acesso em: 19 fev. 2019.

DIAS, Henrique Sant'Anna; LIMA, Luciana Dias de; TEIXEIRA, Márcia. A trajetória da política nacional de reorientação da formação profissional em saúde no SUS. Cien Saude Colet, Rio de Janeiro, v. 18, n. 6, p. 1613-1624, 2013. Disponível em: https://www.scielo.br/pdf/csc/v18n6/13.pdf. Acesso em: 21 jun. 2020.

DOURADO, Carla Solange de Melo Escórcio; COELHO, Maria do Socorro Rodrigues. Adequação dos cursos de Farmácia as novas Diretrizes Curriculares. Revista Científica da FSA, Teresina, v. 7, n. 1, p. 129-142, 2010. Disponível em: http://www4.unifsa.com.br/revista/index.php/fsa/article/view/412. Acesso em: 14 jan. 2019.

FARHAT, Eleide Margarete Pereira et al. Programa de reorientação da formação em saúde relatos de experiências, ações da UNIVALI no Programa de Reorientação da formação em saúde: pró-saúde e pet-saúde. In: FARHAT, Eleide Margarete Pereira; DITTRICH, Maria Glória. Educação e Saúde: Políticas Públicas e Vivências Dialógicas. Itajaí: Ed. da Universidade do Vale do Itajaí, 2016. p. 173-187.

FRANÇA FILHO, José Benedito et al. Perfil dos farmacêuticos e farmácias em Santa Catarina: indicadores de estrutura e processo. Rev. Bras. Cienc. Farm., São Paulo, v. 44, n.1, p. 105-113. jan./mar. 2008. Disponível em:

https://www.scielo.br/pdf/rbcf/v44n1/a12v44n1.pdf. Acesso em: 21 jun. 2020.

GONÇALVES, Daniel Infante Ferreira. Pesquisas de marketing pela internet: as percepções sob a ótica dos entrevistados. Revista de Administração Mackenzie, São Paulo, v. 9, n. 7, nov./dez. 2008.

LIMA, Leonardo Araújo; ANDRIOLA, Wagner Bandeira. Acompanhamento de egressos: subsídios para a avaliação de Instituições de Ensino Superior (IES). Avaliação, Campinas; Sorocaba, v. 23, n. 1, p. 1104-125, 2018. Disponível em:

https://www.scielo.br/pdf/aval/v23n1/1982-5765-aval-23-01-00104.pdf. Acesso em: 28 out. 2020.

MATOS, Izabella Barison; TOASSI, Ramona Fernanda Ceriotti; OLIVEIRA, Maria Conceição de. Profissões e ocupações de saúde e o processo de feminização: tendências e complicações. Athenea Digital, Lages, v. 2, n. 13, p. 239-244, jul. 2013. Disponível em: https://www.lume.ufrgs.br/bitstream/handle/10183/118035/000894801.pdf?sequence=1. Acesso em: 21 jun. 2020.

OLIVEIRA, Naira Villas Boas Vidal de; SZABO, Iolanda; BASTOS, Luiza Lemos; PAIVA, Sabrina Pereira. Atuação profissional dos farmacêuticos no Brasil: perfil sociodemográfico e dinâmica de trabalho em farmácias e drogarias privadas. Saúde soc., São Paulo, v. 26, n. 4, p. 
1105-1121, out./dez. 2017. Disponível em: https://www.scielo.br/pdf/sausoc/v26n4/19840470-sausoc-26-04-1105.pdf. Acesso em: 20 out. 2020.

PEDUZZI, Marina; AGRELI, Heloise Fernandes. Trabalho em equipe e prática colaborativa na Atenção Primária à Saúde. Interface, Botucatu, v. 22, supl. 2, 2018.

Disponível em: https://www.scielo.br/pdf/icse/v22s2/1807-5762-icse-22-s2-1525.pdf. Acesso em: 26 out. 2020.

PEREIRA, Leonardo Régia Leira; FREITAS, Osvaldo de. A evolução da Atenção Farmacêutica e a perspectiva para o Brasil. Revista brasileira de ciências farmacêuticas, São Paulo, v. 44, n. 4, p. 601-612, out./dez. 2008. Disponível em: https://www.scielo.br/scielo.php?pid=s151693322008000400006\&script=sci_arttext\&tlng=pt. Acesso em: 14 set. 2020.

RECINE, Elisabetta et al. Formação profissional para o SUS: análise de reformas curriculares em cursos de graduação em nutrição. Avaliação, Campinas, Sorocaba, SP, v. 23, n. 3, p. 679697, nov. 2018. Disponível em: https://www.scielo.br/pdf/aval/v23n3/1982-5765-aval-23-03679.pdf. Acesso em: 3 maio 2019.

SERAFIN, Claudia; CORREIA JÚNIOR, Daniel; VARGAS, Mirella. Perfil do farmacêutico no Brasil. Brasilia-DF: CFF, 2015. Disponível em: http://www.cff.org.br/userfiles/file/Perfil\%20do\%20farmac\%C3\%AAutico\%20no\%20Brasil \%20_web.pdf. Acesso em: 28 jan. 2019.

SILVA, Elice Maria da et al. Perfil dos egressos de Farmácia de uma Faculdade de Saúde. Revista Infarma, Brasília, v. 31, n. 4, p.259-270, 2019. Disponível em: http://revistas.cff.org.br/?journal=infarma\&page=article\&op=view\&path\%5B \%5D=2519\&pa th\%5B\%5D=pdf. Acesso em: 21 jun. 2020.

SILVA, Roberta de Faria; TORMIN, Consuelo Vaz; PAULA, Victor Gomes de. Percepção de graduandos do curso de farmácia sobre a atuação profissional no mercado farmacêutico. In: SIMPÓSIO, 2019, Luziânia, Goiás. Anais [...]. Luziânia, Goiás: Centro Universitário UNIDESC, 2019. Disponível em:

http://unidesc.web2445.uni5.net/anais_simposio/arquivos_up/documentos/artigos/da2e5f9c84 dab008356dd664cb0ec33d.pdf. Acesso em: 20 out. 2020.

SILVA, Rinaldo Henrique Aguilar da; MIGUEL, Soraida Sozzi; TEIXEIRA, Luciana Scapin. Problematização como método ativo de ensino-aprendizagem: estudantes de farmácia em cenários de prática. Trabalho, Educação e Saúde, Rio de Janeiro, v. 9, n. 1, p. 77-93, mar./jun. 2011. Disponível em: https://www.scielo.br/pdf/tes/v9n1/v9n1a06.pdf. Acesso em: 21 jun. 2020.

SPADA, Celso; CHAGAS, Jair Ribeiro; SILVA, Kátia Flávia Fernandes; CASTILHO, Selma Rodrigues de. A trajetória dos cursos de graduação na Saúde 1991-2004. Brasília, DF:

INEP, 2006. Disponível em: http://portal.inep.gov.br/informacao-da-publicacao/-/asset_publisher/6JYIsGMAMkW1/document/id/489343. Acesso em: 28 jan. 2019.

VIANA, Simone Beatriz Pedrozo et al. Reorientação da formação dos profissionais de saúde com foco na integralidade: a experiência da UNIVALI. In: VIANA, Simone Beatriz Pedrozo; FARHAT, Eleide Margarethe Pereira (orgs.). Experiências inovadoras na formação para o SUS. Curitiba: Appris, 2018. p. 15-35. 